\title{
Structural and functional alterations in the retrosplenial cortex following neuropathic pain
}

\author{
David André Barrière ${ }^{a, b, \star}$, Al Mahdy Hamieh ${ }^{a}$, Ricardo Magalhães ${ }^{b, c, d}$, Amidou Traorée , Julie Barbier $^{a}$, \\ Jean-Marie Bonny ${ }^{\mathrm{e}}$, Denis Ardid ${ }^{\mathrm{a}}$, Jérôme Busserolles ${ }^{\mathrm{a}}$, Sébastien Mériaux ${ }^{\mathrm{b}}$, Fabien Marchand ${ }^{\mathrm{a}}$
}

\begin{abstract}
Human and animal imaging studies demonstrated that chronic pain profoundly alters the structure and the functionality of several brain regions. In this article, we conducted a longitudinal and multimodal study to assess how chronic pain affects the brain. Using the spared nerve injury model which promotes both long-lasting mechanical and thermal allodynia/hyperalgesia but also painassociated comorbidities, we showed that neuropathic pain deeply modified the intrinsic organization of the brain functional network 1 and 2 months after injury. We found that both functional metrics and connectivity of the part A of the retrosplenial granular cortex (RSgA) were significantly correlated with the development of neuropathic pain behaviours. In addition, we found that the functional RSgA connectivity to the subiculum and the prelimbic system are significantly increased in spared nerve injury animals and correlated with peripheral pain thresholds. These brain regions were previously linked to the development of comorbidities associated with neuropathic pain. Using a voxel-based morphometry approach, we showed that neuropathic pain induced a significant increase of the gray matter concentration within the RSgA, associated with a significant activation of both astrocytes and microglial cells. Together, functional and morphological imaging metrics of the RSgA could be used as a predictive biomarker of neuropathic pain.
\end{abstract}

Keywords: Brain, Functional MRI, Imaging biomarker, Neuropathic pain, Retrosplenial cortex, Voxel-based morphometry

\section{Introduction}

With a prevalence of $7 \%$ to $10 \%$ in the general population and a huge societal cost, neuropathic pain is one of the most debilitating painful conditions. ${ }^{16}$ Clinical features of neuropathic pain include pain-evoked response to painful and nonpainful mechanical and cool/hot stimuli, called mechanical and thermal allodynia/hyperalgesia, respectively. Neuropathic pain is also often associated with comorbidities such as anxiety/stress, depression, and cognitive impairments, and to date, numerous studies have shown that neuropathic pain and comorbidities may induce brain reorganization. ${ }^{2,23,49}$

The development of neuroimaging techniques that provide in vivo information of both brain structure and function

\footnotetext{
$\overline{\text { Sponsorships or competing interests that may be relevant to content are disclosed }}$ at the end of this article.

a Inserm U1107 NEURO-DOL, Pharmacologie fondamentale et clinique de la douleur, Université Clermont Auvergne, Clermont-Ferrand, France, ${ }^{b}$ Unité d'imagerie par résonance Magnétique et spectroscopie CEAVDRF/I2BM/ NeuroSpin, Gif-Sur-Yvette, France, ${ }^{\circ}$ Life and Health Sciences Research Institute (ICVS), School of Health Sciences, University of Minho, Braga, Portugal, ${ }^{a}$ ICVS/ 3B's-PT Government Associate Laboratory, Guimarães, Braga, Portugal, ${ }^{e}$ INRA, Centre Auvergne-Rhône Alpes, platforme Agroresonance, Saint Genès Champanelle, France

*Corresponding author. Address: UMR Inserm/UCA 1107, NeuroDol Laboratoire de Pharmacologie Fondamentale et Clinique de la Douleur, BP 10448, F-63000 Clermont-Ferrand, France. Tel.: 0033473178 231. E-mail address: david.a.barriere@gmail.com (D.A. Barrière).
}

Supplemental digital content is available for this article. Direct URL citations appear in the printed text and are provided in the HTML and PDF versions of this article on the journal's Web site (www.painjournalonline.com).

PAIN 00 (2019) 1-14

(C) 2019 International Association for the Study of Pain

http://dx.doi.org/10.1097/j.pain.0000000000001610 revolutionized the investigation of pain perception in human ${ }^{31}$ and animal research. ${ }^{13,14}$ During the last 30 years, numerous MRI data collected in humans and rodents reported a set of structures that systematically respond to noxious stimulations, including the primary (S1) and secondary (S2) somatosensory cortices, the insula, the anterior cingulate cortex, the thalamus, and the prefrontal cortex (PFC). ${ }^{1,14}$ These brain structures, called "the pain matrix," have been previously considered to form a specific network, which could be used as an objective measure of pain, an evidence of pain perception in unconscious patients or an effective biomarker for pain-killer development. ${ }^{12,43,63}$ However, the activation of the pain matrix by nonsensory inputs ${ }^{33,41}$ or in patients with congenital insensitivity to pain ${ }^{58}$ have questioned its specificity. ${ }^{25}$ Recently, a large body of evidence showed that pain should be rather considered as a dynamic experience encoded by a brain network, which integrate cognitive, affective, and sensory aspects of pain. ${ }^{31}$ Thus, neuroimaging based on restingstate functional MRI ( $r s-f M R I)$ with pain threshold assessment before scanning could allow to identify brain networks that correlate with both patients ${ }^{22}$ and animals pain thresholds. ${ }^{9}$ Indeed, since brain continuously encodes and processes spontaneous pain, rs-fMRI method provides a powerful tool to detect the functional brain dynamic and reorganization related to chronic pain. For example, functional connection changes were localized mainly within the limbic system, as well as between the limbic and nociceptive systems in the spared nerve injury (SNI) model, ${ }^{9}$ which were correlated with mechanical allodynia. In another hand, human and rodent studies have also demonstrated large-scale brain morphological reorganization during chronic pain. Volumetric reductions in the rat PFC and other cortical regions, including the anterior cingulate cortex, primary somatosensory cortex (S1), and insula were observed as late as 4 months after SNI. ${ }^{28,60,62,66}$ These structural changes have been 
correlated with mechanical hypersensitivity development and interestingly, to the emergence of anxiety-like behaviours.

Nevertheless, the relationship between brain structural and functional modifications with pain behaviours and especially, associated emotional/cognitive alterations in animals is still missing. Our aim was to combine structural and functional MRI, pain and emotional/cognitive behavioural assessment to identify the brain territories and brain functional impairments involved in neuropathic pain. Furthermore, we investigated neuronal and glia activation within identified brain structures using an immunohistochemical approach.

\section{Methods}

\subsection{Animals}

All experiments were performed using male Sprague-Dawley rats (150-175 g; Charles River, Lyon, France). Animals were housed 4 per cage under standard conditions $\left(21-22^{\circ} \mathrm{C}\right.$; $12 / 12$ hours light/ dark cycle; 55\% humidity) with food and water ad libitum during 1 week before experiments to allow for acclimatization. Experiments followed guidelines of the Committee for Research and Ethical Issues of IASP ${ }^{75}$ and conducted in strict accordance with the recommendations of the European Community (2010/63/ EU), the French legislation (decree no 2013-118) for use and care of laboratory animals, and were locally approved by the regional ethics committee for animal experimentation (CEMEA Auvergne, France).

\subsection{Experimental design}

A first cohort of 24 animals was used, 12 animals for the SNI group, and 12 animals for the sham group. Both pain thresholds and brain imaging were investigated in 2 different groups of 6 Sham and $6 \mathrm{SNI}$ animals, randomly chosen, for each time point (ie, days 30 [1 month group] and days 60 [2 months group]) after surgery. Each animal of each group has been scanned once. Three rats (one SNI animal at day 30 and 2 Sham animals at day 30 and 60) were excluded from analysis because of excessive motion during imaging acquisition, $\left(n_{\text {(final })}=21\right)$. At the end of each scanning session, anesthetized animals were sacrificed immediately, and brains were collected for immunohistochemistry (see immunohistochemistry section). After scanning results, a second cohort of 40 animals was used to assess both spatial and social memory ( $Y$-maze and social recognition memory tests, respectively) and response to aversive stimulation (contextual fear conditioning). Twenty animals were Sham-operated, and 20 were $\mathrm{SNI}$ animals. Ten sham and $10 \mathrm{SNI}$ animals were randomly selected for the different behavioural tests ( $\mathrm{Y}$-maze, social recognition test, fear conditioning) 30 days ( 1 month group) and 60 days ( 2 months group) after surgery (experimental design summary in Fig. 1A).

\subsection{Spared nerve injury model}

A previously described SNI pain model was used. ${ }^{17}$ Briefly, rats were anesthetized with a mixture of ketamine $(75 \mathrm{mg} / \mathrm{kg})$ and xylazine $(10 \mathrm{mg} / \mathrm{kg})$. The common sciatic nerve was exposed at the trifurcation level by blunt dissection through the biceps femoris. The right common peroneal and the tibial nerves were exposed, tight-ligated with one ligature of 5.0 silk, and then sectioned distal to the ligation, removing around 3 to $4 \mathrm{~mm}$ of the distal nerve stump. Great care was taken to avoid any contact or stretching with the sural nerve. Muscle and skin were closed with
3.0 suture. During the following week after surgical procedure, animals were visited daily, weighted, and observed. We performed the SNI procedure on the right sciatic nerve since rats exhibit cognitive deficits and depressive-like behaviours in addition to pain but present normal anxiety level in contrast to the left sciatic nerve injury, which leads mainly to anxiety-like behaviour. $^{36}$ Indeed, we did not find a significant difference between SNI and Sham rats in the elevated plus maze test 1 month after surgery (data not shown).

\subsection{Assessment of pain thresholds}

Both mechanical and cold sensitivity of Sham and SNI rats were assessed 1 and 2 months after surgery, 1 day before scanning. Mechanical sensitivity was tested using von Frey filaments. Animals were acclimatised during 30 minutes in individual clear acrylic cubicle $(22 \times 16.5 \times 14 \mathrm{~cm})$ and placed on the top of an elevated wire mesh. Each von Frey filaments of $4 \mathrm{~g}, 8 \mathrm{~g}$, and $15 \mathrm{~g}$ were applied 5 times on the right hind paw. Quick withdrawal or licking of the paw to the stimulus was considered as a positive response, and results are expressed as the percentage of positive response \pm SEM to the mean obtained for each von Frey filament. ${ }^{19}$ Cold sensitivity (acetone test) was performed as described by ${ }^{15}$ using a syringe connected to a PE-90 tubing; a drop of acetone was applied to the plantar surface of the right hind paw. The pain-related behaviour evoked by the cooling of acetone was graded to the following 4-point scale: $0=$ no response; 1 = quick withdrawal, flick or stamp of the paw; $2=$ prolonged withdrawal or repeated flicking of the paw; $3=$ repeated flicking and licking of the paw. Acetone was applied 6 times with 5-minute interval between each successive application. Results are expressed as the total score following the 6 applications (maximum score $=18$ ).

\subsection{Animal preparation for MRI experiments}

The day after pain behavioural assessment, rats were anaesthetized using isoflurane (3\%-4\%, in 30\% $\mathrm{O}_{2} / 70 \%$ air mix) then, transferred to a cradle dedicated to rat brain MRI, and supplied with a $1 \%$ to $2 \%$ isoflurane in $30 \% \mathrm{O}_{2} / 70 \%$ air mix using a fitted mask. Body temperature was maintained at $37^{\circ} \mathrm{C}$ using a feedback-controlled heated water/air system using a rectal probe (thermocouple, Eurotherm, France). Breath rate was also recorded throughout the MRI experiment (Windaq software; DataQ instruments, Akron, $\mathrm{OH}$ ) and used for noise regression (see fMRI data preprocessing section).

\subsection{Scanning procedure}

$\mathrm{MRI}$ experiments were performed on the MRI facility (Plateforme AgroResonance; INRA, Saint Gènes Champanelle, France) at 11.7 Tesla (BioSpec 117/16 preclinical scanner, Bruker; Ettlingen, Germany). A 72-mm diameter volume coil was used for RF transmission, and a 4-channel phased array surface coil was used for signal reception. After positioning, a first set of rs-fMRI data was acquired during 13 minutes using a 2-shot 2D SE-EPI sequence $(\mathrm{TE} / \mathrm{TR}=17.5 / 650 \mathrm{~ms}$, segments $=2$, field of view $=$ $30 \times 30 \times 12 \mathrm{~mm}^{3}$, 1-mm slice thickness, acquisition matrix $=$ $96 \times 96 \times 12$ for a final resolution image of $312 \times 312 \times$ $1000 \mu^{3}$ ) corresponding to 600 volumes covering the middle of the olfactory bulb to the middle of cerebellum for studying changes in brain functional connectivity. During the functional acquisition, breath rate and body temperature were monitored and maintained continuously around $70 \mathrm{BPM}$ and $37^{\circ} \mathrm{C}$ to 


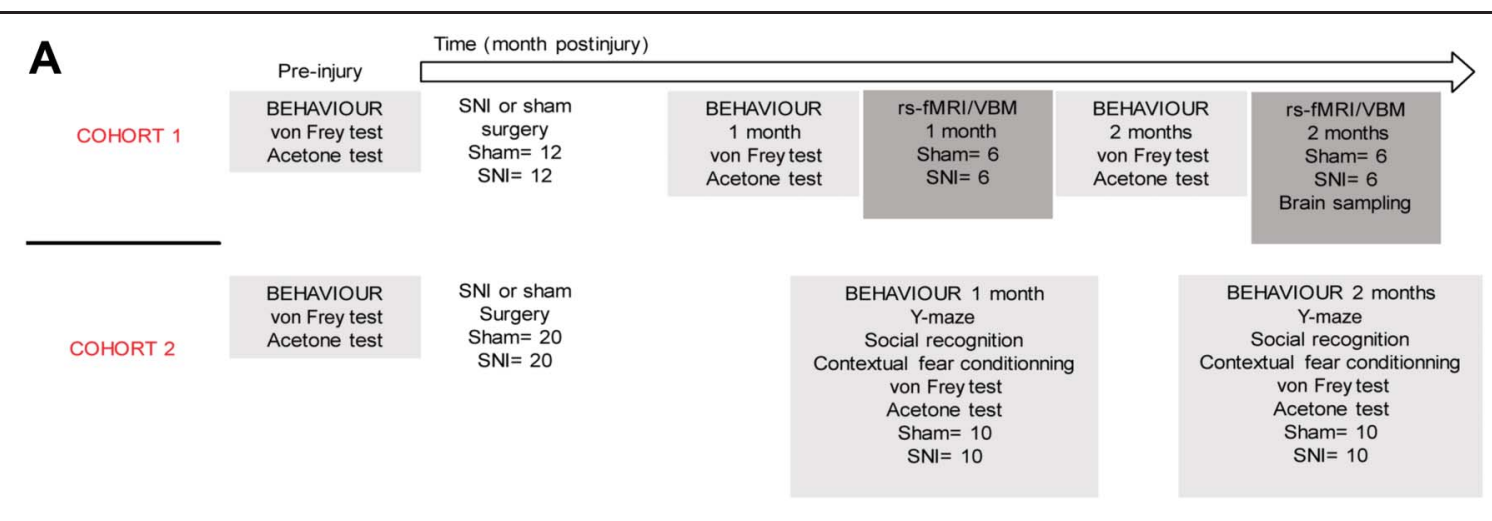

B

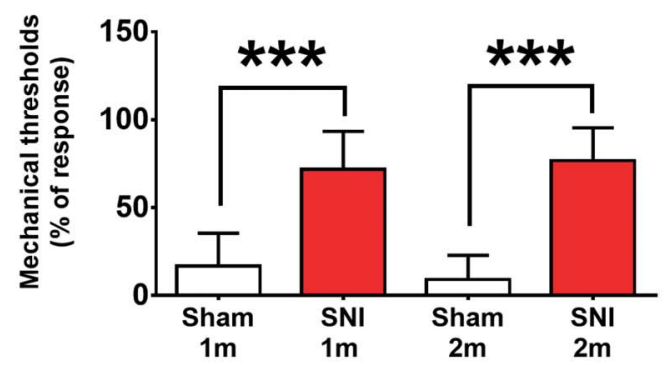

D

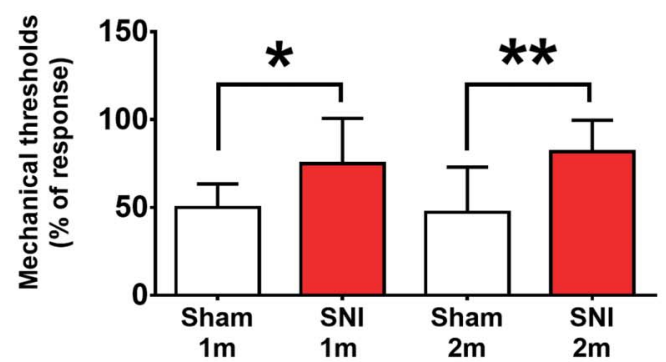

C

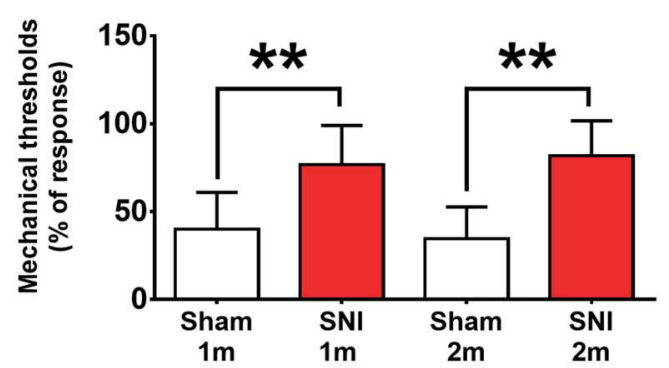

E

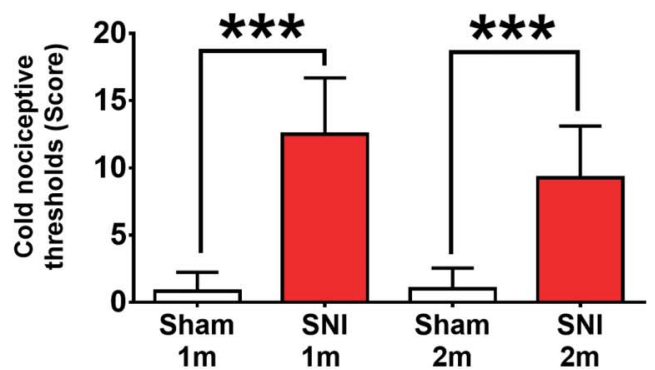

Figure 1. Effect of spared nerve injury on mechanical thresholds and cold scores. (A) Summary of the experimental design. In these experiments, we observed a significant decrease of mechanical threshold using the $4 \mathrm{~g}(\mathrm{~B}), 8 \mathrm{~g}(\mathrm{C})$, and $15 \mathrm{~g}(\mathrm{D})$ von Frey hairs indicating the development of mechanical allodynia and hyperalgesia at 1 and 2 months after surgery. Moreover, we observed the development of cold allodynia using the acetone test (E). Data were compared using on rank one-way ANOVA followed by Holm-Sidak post hoc test. Error bars represent the SEM. ${ }^{\star} P<0.05,{ }^{\star \star} P<0.01$, ${ }^{\star \star \star} P<0.001$ compared with the Sham group $(\mathrm{n}$ $=5-6$ per group and time point). ANOVA, analysis of variance.

minimize the possible effects of anaesthesia (Fig. S1, available at http://links.lww.com/PAIN/A805). After the functional restingstate scanning procedure, an anatomical image was acquired using a $T_{2}$-weighted RARE sequence (Turbo-RARE Factor $=4$, $\mathrm{TE} / \mathrm{TR}=10 / 4600 \mathrm{~ms}, 1$ repetition, field of view $=32 \times 32 \times$ $27 \mathrm{~mm}^{3}, 0.5-\mathrm{mm}$ slice thickness, acquisition matrix $=256 \times$ $256 \times 54$ slices for a final resolution image of $125 \times 125 \times$ $500 \mu^{3}$ ) covering the entire brain. Both functional and anatomical MRI data were reconstructed using homemade Matlab routines (The Mathworks) and saved in Nifti format for analysis.

\subsection{Rat brain template and atlas}

Anatomical images were segmented and co-registered to an inhouse anatomical rat brain template and its corresponding atlas previously used in. ${ }^{42}$ Our high-resolution template $(0.09 \mathrm{~mm}$ isotropic voxels) was created using ex vivo $T_{2}{ }^{*}$-weighted images acquired from 16 rats using the SPM8 unified segmentation approach to create gray matter (GM), white matter (WM), and cerebrospinal fluid (CSF) rat brain tissue priors used to perform both rs-fMRI and voxel-based morphometry (VBM) analysis here (Fig. S2, available at http://links.Iww.com/PAIN/A805). Our inhouse built rat brain atlas used here results from the normalisation of existing rat brain atlases ${ }^{48,69}$ to our template image.

\subsection{Resting-state fMRI data preprocessing}

Resting-state $\mathrm{fMRI}$ data sets were preprocessed with SPM8 using the SPM mouse 1.1 Toolbox ${ }^{60,61}$ running on Matlab 2017 a (Mathworks) and FSL 5.1 software. In the first step of preprocessing, EPI images were resized by a factor of 10 , aligned to our rat brain stereotaxic space, slice-time corrected, realigned, and resliced to the first volume, and coregistered with their corresponding anatomical images using SPM8. Next, anatomical images were segmented using our rat brain tissue priors, first, to create specific brain masks for each anatomical image (GM + WM + CSF = Brain Mask) and, second, to 
normalize both anatomical and functional images onto our brain template using the affine matrix calculated by SPM8 during the segmentation step. Finally, normalized images were masked to remove soft tissue using the brain masks generated previously and spatially smoothed with a Gaussian kernel of 6-mm FWHM. In the second step of preprocessing, EPI images were processed using FSL 5.1. First, the effect of the previously calculated 6 motions parameters, including translations and rotations, out brain signal and breathing parameters recording during the acquisition were removed from the data through linear regression using the FEAT procedure and the residuals kept as signal of interest. Then, acquisition artefacts (ghosting) were identified using the melodic function. Briefly, melodic decomposes the signal of the $4 \mathrm{D}$ smoothed images into independent spatial components. These components were individually thresholds, visually inspected and the once identified as artefacts were removed using the regfilt function of FSL. ${ }^{30,42}$ Finally, a band pass filtering $(0.1-0.005 \mathrm{~Hz})$ was applied to the time series data (Fig. S2, available at http://links.Iww.com/PAIN/A805).

\subsection{Construction of resting-state functional connectivity networks}

Each 4D processed functional images (21 data sets) were segmented into 120 anatomical region of interest (ROI) (60 regions in each hemisphere, Table S1, available at http://links. Iww.com/PAIN/A806; title available at http://links.Iww.com/ PAIN/A805) using the GM ROls of our rat atlas and the REX function of the CONN toolbox. ${ }^{71}$ For each animal, functional correlation coefficients ( $r$ values) were computed between the time course of each pairs of ROls and then transformed to zscores using Fisher's $z$ transformation, resulting in a $120 \times 120$ matrix of normalized correlation coefficients for each animal. Networks of altered connectivity were computed, extracted, and plotted using the Network-Based Statistic (NBS), ${ }^{73}$ the Brain Connectivity Toolbox, ${ }^{57}$ the BrainNet Viewer toolbox, ${ }^{72}$ and inhouse developed Matlab scripts (Mathworks). Statistical comparisons of FC (functional connectivity) between groups were made at the global network level (ie, conjunction of all ROIs) using NBS. The NBS methodology works in 2 steps: first, the statistical hypothesis was tested at each connection and its statistical significance determined. Second, the connections were thresholded by a user-defined significance, the subnetwork components (groups of nodes and edges such that a path can be found between any pair of network members) were found and their size (number of surviving connections) determined. The component significance was determined through permutation testing, where the tested subjects are randomly permutated (5000 permutations) between groups, and the chance of randomly finding networks of similar size determined, outputting a family-wise error rate corrected significance $\left(\alpha_{\mathrm{FWE}}, 0.05\right)$. Statistical correlation between pain scores and functional connectivity were assessed by using 2 tailed $t$ test with the following incremental thresholds for $P=0.01\left(\mathrm{t}_{(19)}=2.85\right), P=0.005\left(\mathrm{t}_{(19)}=3.17\right)$, and $P=0.001$ $\left(t_{(19)}=3.88\right)$.

\subsection{Brain metrics calculation}

Global and local network measures including global efficiency, transitivity, global clustering, and small worldness were computed using the CONN toolbox in Matlab (Fig. S2, available at http://links.Iww.com/PAIN/A805). To identify the nodes of the network that are the most involved in the pain-affected networks as well as the most affected connections, local measures such as betweenness centrality, local clustering, and cost have been calculated using the CONN toolbox. ${ }^{71}$

\subsection{Voxel-based morphometry data preprocessing}

Voxel-Based Morphometry data were preprocessed with SPM8 using the SPM mouse 1.1 Toolbox. ${ }^{60,61}$ Each $T_{2}$-weighted anatomical image was resized by a factor of 10 , aligned to the stereotaxic space by registering each image to our rat brain template image using SPM mouse. Then, each image was bias corrected and segmented into probability maps of GM, WM, and CSF using our rat brain priors (Fig. S2, available at http://links. Iww.com/PAIN/A805). Each GM, WM, and CSF probability map were normalized to our stereotaxic space using transformations matrices obtained herein and resampled to $1.25 \times 1.25 \times$ $1.25 \mathrm{~mm}^{3}$ (in resized images). Normalized GM and WM images were used to create a more population-specific template using diffeomorphic anatomical registration using exponentiated lie algebra (DARTEL). ${ }^{5-7}$ Each normalized GM image was then warped using deformation parameters calculated by the DARTEL routine of SPM8 and then modulated to correct the volume changes that may have occurred during the deformation step. Finally, normalized-warped-modulated GM images were spatially smoothed with an isotropic Gaussian kernel to create gray matter concentration (GMC) maps by convolving a 6-mm full width at half maximum. Brain's volume of each animal was estimated from the initial GM, WM, and CSF images generated by the first segmentation processing. Gray matter, WM, and CSF images were added and thresholded to create brain mask images then positive voxels were counted, and volume of the entire brain was determined from the initial resolution (Fig. S2, available at http:// links.Iww.com/PAIN/A805).

\subsection{Voxel-based morphometry statistic and analysis}

To assess regional GM changes in SNI animals, their GMC maps were compared to those obtained from Sham rats using a 2sample $t$ test. Even if we did not observe any significant variation in the brain volume between Sham and SNI groups, we observed a significant increase in brain volume between the first and the second months in both groups (Sham 1 and 2 months: $2.034 \mathrm{~mL}$ $\pm 0.001 / 2.184 \mathrm{~mL} \pm 0.002, P<0.01$ and SNI 1 and 2 months: $1.992 \mathrm{~mL} \pm 0.001 / 2.118 \mathrm{~mL} \pm 0.002, P<0.05$; one-way analysis of variance, Holm-Sidak post hoc test). Hence, to avoid age-dependent confounding effect, we added brain volume as a confounding factor in our statistical image analysis. The significance level applied to GMC images was set at $P<0.02$ $\left(t_{(19)}=2.213\right.$, FDR-corrected), with a minimum cluster size set at 200 voxels.

\subsection{Behavioural assessments}

\subsubsection{Social recognition memory test}

The procedure used was adapted from Perio et al. ${ }^{50}$ Briefly, SNI and Sham rats were individually housed for 2 days before testing. On the testing day, a juvenile rat (40-60 g), after 20 minutes of isolation, was introduced into the SNI or sham rats' cage for 10 minutes. The time spent in active social interaction (ie, time spent by the adult rat sniffing, following, biting, jumping, and crawling over or under the juvenile) during the first 5 -minute period (T1) and the second 5-minute period (T2) was counted. The difference between T1 and T2 (social recognition score) was analysed for each adult rat. An animal with a good social memory capacity 
decreases its social interaction toward the juvenile during the second phase (T2), ie, recognition of the young animal, and therefore presents a high social recognition score. By contrast, a low social recognition score means that the adult rat continues to interact with the juvenile during $\mathrm{T} 2 .^{50}$

\subsubsection{Spatial recognition memory test ( $Y$-maze)}

The $Y$-maze test was adapted from Dellu et al. ${ }^{18}$ The $Y$-maze apparatus consists of 3 identical arms $(45 \times 45 \times 13 \mathrm{~cm})$ equally distributed around a central triangle. Visual cues were placed on the walls of the experimental room. The task consisted of 2 trials with a 2-minute intertrial interval. At the beginning of the test, each animal was placed into the central triangle, always facing the same arm. Rats were then allowed to explore 2 arms during 5 minutes while one arm was physically blocked (novel arm). In the second trial, rats were allowed to freely explore all 3 arms for 5 minutes. Total number of entries and the percentage of time spent in each arm were recorded and manually scored. Arm entry was defined as all 4 paws into the arm. A decrease in the percentage of time spent in the novel arm reflects spatial memory cognitive impairments. ${ }^{18}$ Spared nerve injury and Sham animals were tested 1 and 2 months after surgery.

\subsubsection{Contextual fear conditioning}

Fear response of SNI and Sham animals was evaluated using the contextual fear conditioning test. The apparatus consists of a conditioning chamber equipped with a grid floor of stainless steel bars connected to a shock generator used for the delivery of a foot shock (Imetronic, France). Conditioning chambers were cleaned using $70 \%$ ethanol following use. On the training session, the rat was placed in the conditioning chamber and allowed to freely explore for a 3-minute habituation period followed by one single shock (1 mA, 2-second duration) and then retained in the context for 1 minute after the shock administration. One day after training, contextual fear conditioning was evaluated by exposing the rat to the same conditioning chamber during 6 minutes without any shock delivery. Rat's behaviour was recorded during both training and testing sessions using a video camera positioned in front of the chamber. The assessment of fear was performed by measuring the freezing time, which is defined as complete immobility of the animal except of breathing movements for a period of at least 2 seconds. Time spent in freezing for each rat was manually scored by a blind experimenter. The amount of freezing was expressed as a percentage of the total scoring period.

\subsection{Immunohistochemistry}

Two months after surgery, SNI and Sham animals $(n=6$ in each group) that underwent VBM and rs-fMRI imaging, were terminally anaesthetised using pentobarbitone and quickly perfused transcardially with saline followed by $4 \%$ paraformaldehyde. After perfusion, the brain was excised, postfixed for 24 hours in the same perfusion fixative, cryoprotected in $30 \%$ sucrose in $0.1-\mathrm{M}$ phosphate buffer for 48 hours at $4^{\circ} \mathrm{C}$, and then frozen in optimum cutting temperature freezing medium. Transverse sections (30 $\mathrm{mm}$ ) were cut on a cryotome (Microm HM450; Thermo Scientific, Illkirch, France). Free floating sections of the retrosplenial region were double stained for astrocytes (GFAP) and microglia (Iba1) or stained only for NeuN (neurons). Immunohistochemistry has been processed as follows: after 3 washes in TBS $0.05 \mathrm{M}$, Triton $0.3 \%, \mathrm{pH} 7.6$, sections were incubated overnight at room temperature with anti-NeuN ([mouse, antineuronal nuclei]; 1:1000; Millipore, neurons; MerkMillipore, Molsheim, France), or with a mixture of anti-lba1 ([rabbit, antiionized calcium binding adaptor molecule 1]; 1:1500; Wako Pure Chemical Industries Ltd, Osaka, Japan, microglial cells) and antiGFAP ([chicken, antiglial fibrillary acidic protein]; 1:2000; Millipore, astrocytes). After 3 washes in TBS $0.05 \mathrm{M}$, sections were incubated for 2 hours with the appropriate secondary antibody (1: 2000; AlexaFluor 488 goat anti-mouse lgG, AlexaFluor 488 goat anti-chicken lgG or AlexaFluor 546 goat anti-rabbit lgG; Molecular Probes, Thermo Scientific). Sections were then washed in TBS $0.05 \mathrm{M}$, mounted on gelatine-coated slides and cover-slipped with Dako fluorescent mounting medium. Sections were visualised under Nikon Eclipse Ni-E fluorescent microscope with Nikon analysis software (NiSelement). From each animal, 4 to 6 sections were randomly selected for counting and an automatic method was used for NeuN count using threshold analysis. Fluorescent intensity was measured using ROI defined on the RSgA for Iba1 and GFAP staining (4-6 sections of the contralateral side were randomly selected for analysis).

\subsection{Statistical analysis}

All data were analysed using GraphPad Prism 6.02 software. The data are presented as mean \pm SEM. Differences in pain thresholds mean connectivity and RSgA GMC were evaluated using a one-way repeated-measures analysis of variance, followed by Holm-Sidak post hoc test. Data from correlation were estimated using a two-tailed parametric Pearson test. Specificity and selectivity analysis were performed using the receiver-operating characteristic $(\mathrm{ROC})$ curves method. The level of statistical significance was set at $P<0.05$.

\section{Results}

\subsection{Influence of pain thresholds on brain functional connectome}

One and 2 months after surgery, SNI animals exhibited a significant increase of the percentage of response to the different von Frey filaments, allodynic (4 g, Fig. 1A), intermediate (8 g, Fig. 1B), and hyperalgesic (15 g, Fig. 1C) compared with Sham animals, illustrating the development of mechanical allodynia/hyperalgesia. Moreover, significant cold allodynia, assessed by the acetone test, was observed in SNI animals compared with Sham as previously showed ${ }^{15}$ (Fig. 1D). To evaluate how neuropathic pain modify the brain connectome, we used the mechanical and cold pain scores of each animal as a covariate in our rs-fMRI study to find which brain networks are correlated with these different pain thresholds. Using the pain scores of the allodynic $4 \mathrm{~g}$ von Frey filament, we found that the development of mechanical allodynia in SNI animals was significantly correlated $(R=0.835, P<0.001)$ with an increase of 47 functional connections shared cortically between the granular/agranular/dysgranular insular cortices, lateral and medial parietal associative cortices, orbitofrontal region, prelimbic system, pretectal region, primary and secondary cingulate cortices, primary and secondary motor cortices, primary somatosensory cortex, retrosplenial dysgranular and granular (part a and part b) cortices, and subcortically between the basal forebrain region, bed nucleus of the stria terminalis (BNST), hippocampus, parasubiculum, subiculum, periaqueductal grey (PAG), striatum, superficial gray layer of the superior colliculus, and thalamus (Fig. 2A, Table S2, available at http://links.Iww. com/PAIN/A807—title available at http://links.lww.com/PAIN/ A805). By contrast, using the pain scores of the intermediate 
allodynic/hyperalgesic $8 \mathrm{~g}$ von Frey filament, only 18 functional connections were significantly correlated with these pain scores $(R=0.634, P<0.01)$, which were shared cortically between the granular insular cortex, orbitofrontal region, prelimbic system, primary and secondary motor cortices, primary somatosensory cortex, retrosplenial dysgranular, and granular (part a and part b) cortices and between subcortical areas such as the hypothalamic region and the subiculum (Fig. 2B, Table S3, available at http:// links.Iww.com/PAIN/A808 - title available at http://links.lww.com/ PAIN/A805). Interestingly, using the hyperalgesic $15 \mathrm{~g}$ von Frey filament scores, we found a larger network significantly correlated with these pain scores $(R=0.814, P<0.001)$, composed by 92 functional connections shared cortically between the granular/ agranular/dysgranular insula, ectorhinal and entorhinal, lateral and posterorostral parietal associative region, orbitofrontal region, prelimbic system, primary and secondary cingulate, primary and secondary motor, primary somatosensory, retrosplenial dysgranular, and granular (part a and part b) cortices and between subcortical regions such as the basal forebrain region, hippocampus, interpeduncular nucleus, PAG, striatum, subiculum, superficial gray layer of the superior colliculus and thalamus (Fig. $2 \mathrm{C}$, Table S4, available at http://links.Iww.com/PAIN/A809—-title available at http://links.Iww.com/PAIN/A805). Finally, the development of cold allodynia in SNI animals assessed by the acetone test was significantly correlated $(R=0.813, P<0.001)$ with a different network than the one obtained with the mechanical allodynic network associated with the $4 \mathrm{~g}$ von Frey filament. This network was formed by 27 functional connections shared between cortical areas such as the granular/agranular/dysgranular insula, ectorhinal, secondary motor cortex, lateral and medial parietal associative, prelimbic system, primary and secondary somatosensory, retrosplenial granular (part a and part b) cortices, and with subcortical regions such as the basal forebrain region, hippocampus, hypothalamic region, subiculum, striatum, and the superficial gray layer of the superior colliculus (Fig. 2D, Table S5, available at http:// links.Iww.com/PAIN/A810 — title available at http://links.Iww.com/ PAIN/A805). The global network topological metrics were calculated for both Sham and SNI animals according to Baliki et al., $2014 .{ }^{9}$ In agreement with their results, the global efficiency, the transitivity coefficient, the global clustering coefficient, and the small worldness coefficient were not significantly different between SNI and Sham animals, and no effect of time has been observed (Fig. S4, available at http://links.lww.com/PAIN/A805). Hence, neuropathic pain did not modify the global properties of brain networks but rather reorganized the functional connectivity between critical regions involved in neuropathic processes. To identify these regions, we investigated the local centrality (numbers of significant connections reaching the node) of each node between each animal. First, we found that 12 regions, namely the retrosplenial dysgranular and granular (part a and part b) cortices, the granular/agranular/dysgranular insular cortices, the lateral parietal associative cortex (PtA), the prelimbic system, the striatum, and the superficial gray layer of the superior colliculus were systematically associated with the $4 \mathrm{~g}$ (Fig. 3A), $8 \mathrm{~g}$ (Fig. 3B), $15 \mathrm{~g}$ von Frey filaments (Fig. 3C), and acetone (Fig. 3D) identified networks. Second, the calculation of the centrality for each of those nodes revealed that the retrosplenial regions, especially the contralateral retrosplenial granular part a cortex (Left RSgA), displayed the greatest alteration of the centrality within all networks. This specific increase of the RSgA centrality and its systematic presence within the brain networks correlated with the different pain scores motivated a deeper investigation of its functional connectivity and metrics.

\subsection{Influence of pain thresholds on the RSgA functional connectivity}

To investigate specifically the role of the RSgA in neuropathic pain, we evaluated which functional connections of both left and right RSgA were significantly correlated with $4 \mathrm{~g}, 8 \mathrm{~g}$, and $15 \mathrm{~g}$ von Frey filaments as well as acetone pain scores and the brain structures involved. We found that the contralateral RSgA forms 12 different functional connectivity, which are significantly correlated with all the different pain scores namely, the right lateral PtA, the right orbitofrontal region, the left agranular and granular insular cortex, the left basal forebrain region, the left prelimbic system, the left primary somatosensory cortex barrel field and hind limb areas, the left secondary cingular cortex, the left striatum, the left subiculum, and the left thalamus. In another hand, the right RSgA forms 6 functional connections significantly correlated with all the different pain scores namely, the PAG, the right prelimbic system, the right and left primary cingular cortex, the left primary somatosensory cortex trunk, and the right secondary motor cortex (Fig. 4A and Table S6, available at http://links.lww.com/PAIN/A811-title available at http://links. Iww.com/PAIN/A805). Finally, the correlation analysis of the mean connectivity of this network with mechanical allodynia and hyperalgesia scores as well as cold allodynia scores were significant for each comparison (Fig. 4B).

\subsection{The functional connectivity of $R S g A$ as a putative neuroimaging biomarker of neuropathic pain}

Because the RSgA seems a critical structure involved in the changes of brain functional connectivity following neuropathic pain, we assessed certain behaviours involving the RSgA and its functional links with the prelimbic system and the subiculum regions. ${ }^{35,51,52,68}$ Indeed, these structures have been involved directly and/or indirectly in pain processes and, for some of them, associated comorbidities such as memory impairment, fear response, and depression. ${ }^{44,55}$ We therefore investigated the effect of the SNI procedure on recognition/spatial memory and fear response using a second cohort of Sham and SNI animals in the same conditions as previously described. We observed a significant decrease of both social and spatial memory 1 and 2 months after SNI, assessed by the social recognition (Fig. 5A) and the $\mathrm{Y}$-maze tests (Fig. 5B), respectively, illustrated by a reduction of the social recognition score and a decrease of the percentage spent in the novel arm in the SNI group compared with the Sham group. In addition, a significant decrease of extinction to contextual fear 1 and 2 months after SNI was observed, using the contextual fear conditioning test (Fig. 5C). Sham animals exhibited a reduction of the freezing time following subsequent exposure to context while SNI animals still significantly freeze when exposed to context. Thus, in addition to sensory alterations, both mnesic and fear-related systems seem impaired in the SNI model. Accordingly, we demonstrated that both RSgA, prelimbic region, and RSgA, subiculum functional connections, were significantly increased in SNI animals compared with Sham animals (Fig. 6). Importantly, they were significantly correlated with the $4 \mathrm{~g}$ von Frey score (Fig. 6A), the $8 \mathrm{~g}$ von Frey score (Fig. 6B), the $15 \mathrm{~g}$ von Frey score (Fig. 6C), and the acetone score (Fig. 6D). Using a receiver-operating characteristic $(\mathrm{ROC})$ procedure to estimate sensitivity (ability to detect "neuropathic pain") and specificity (ability to detect "no neuropathic pain") of both RSgA, prelimbic region, and RSgA, subiculum functional connections (Fig. 6E), we demonstrated their potential to predict such type of pain. The ROC analysis 
A

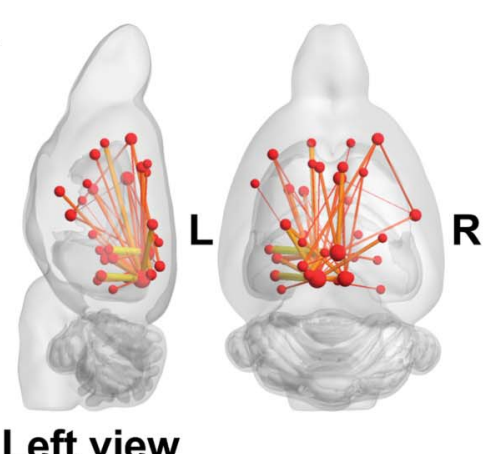

B

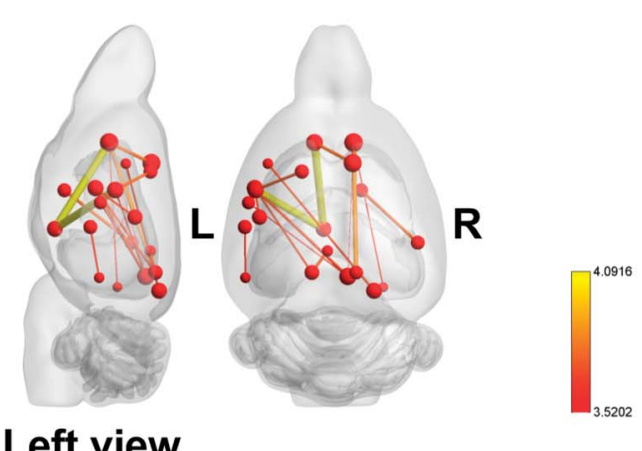

\section{Left view}

C

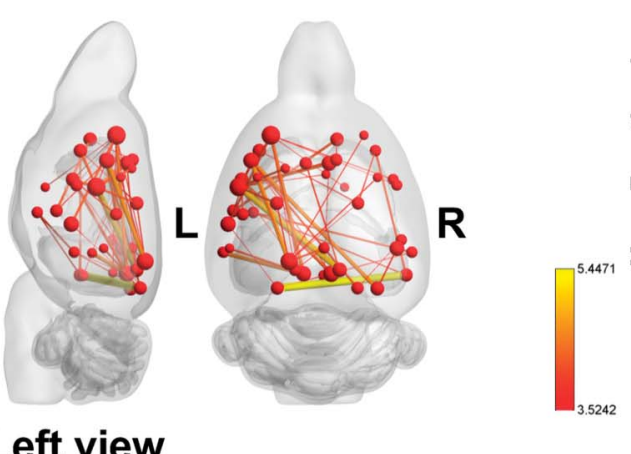

\section{Left view}

D

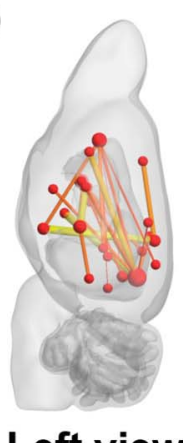

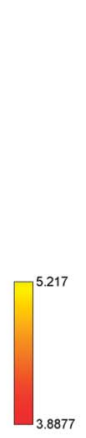
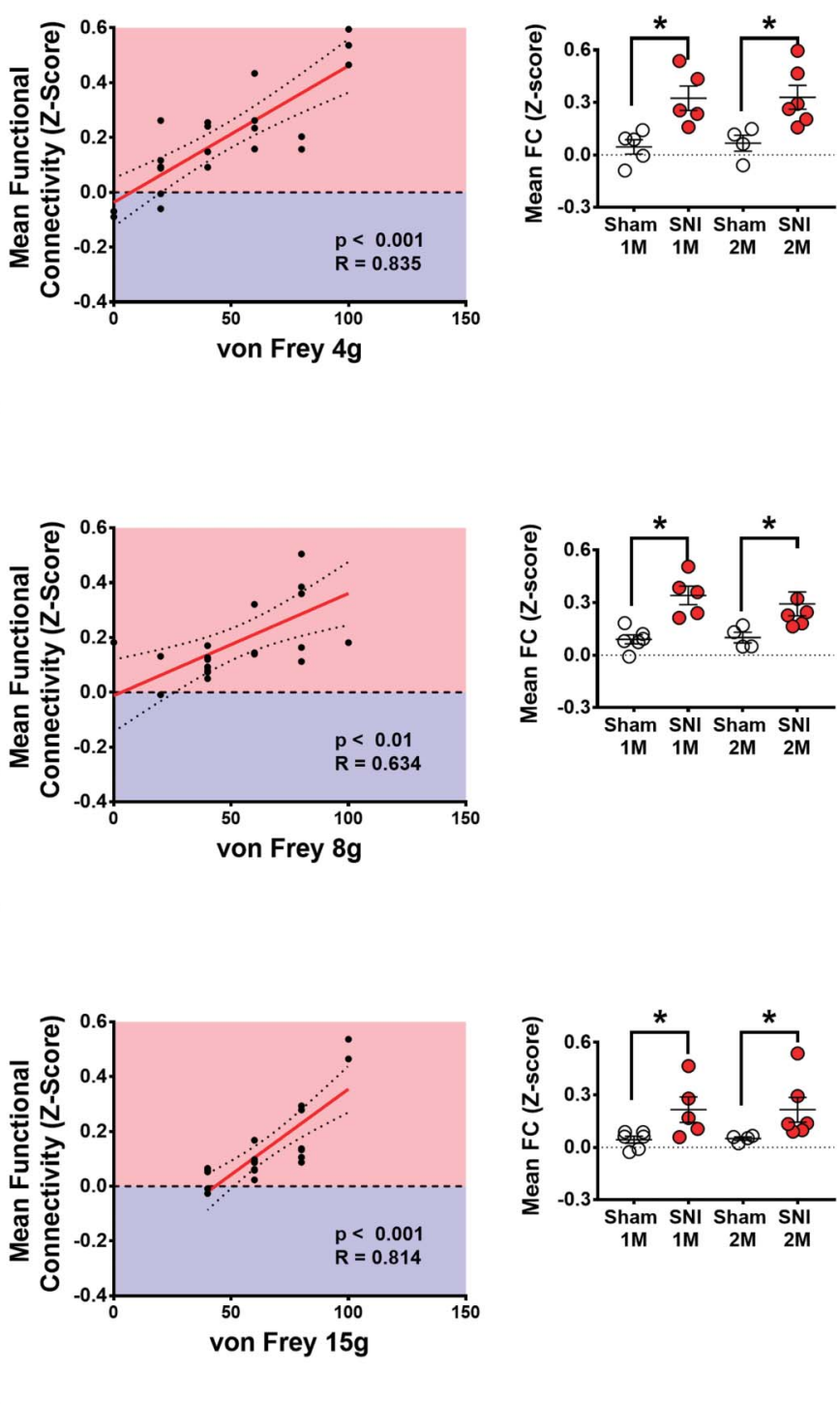

\section{Left view}
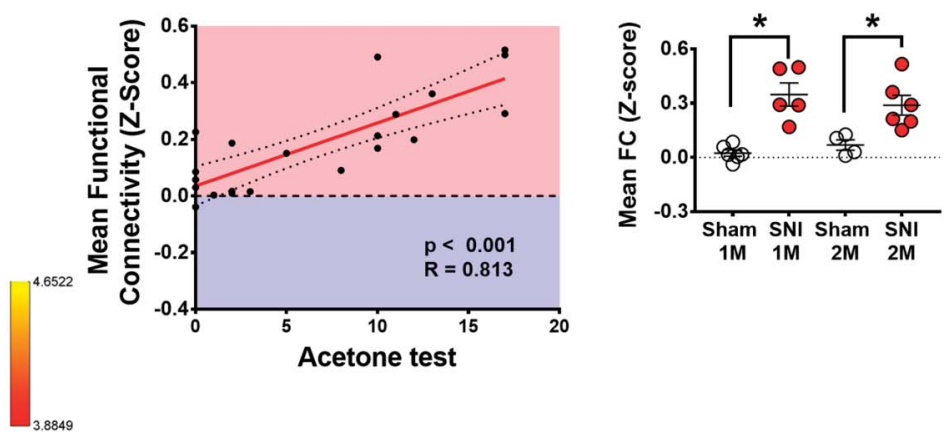

Figure 2. Effect of spared nerve injury on brain connectivity reorganization associated with the pain scores. Specific networks that are significantly correlated with 4 $\mathrm{g}$ von Frey (A), $8 \mathrm{~g}$ von Frey (B), $15 \mathrm{~g}$ von Frey (C), and acetone scores (D). Data were compared using a Network-Based Statistic $t$ test method using 5000 permutations and an extended threshold set at 0.05. von Frey $4 \mathrm{~g}$ network represents 47 positives connections ( $\left.\mathrm{t}_{(19)}=3.88, P<0.001\right)$, von Frey $8 \mathrm{~g}$ network represents 18 positives connections $\left(\mathrm{t}_{(19)}=2.43, P<0.025\right)$, von Frey $15 \mathrm{~g}$ network represents 92 positives connections $\left(\mathrm{t}_{(19)}=3.17, P<0.005\right)$, and acetone network represents 27 positives connections $\left(t_{(19)}=3.88, P<0.001\right)$. Correlation were estimated using a two-tailed parametric Pearson test, and group comparison were estimated using a one-way ANOVA followed by Holm-Sidak post hoc test. Error bars represent the SEM. ${ }^{*}<<0.05$, compared with the Sham group $(n=21)$. ANOVA, analysis of variance. 

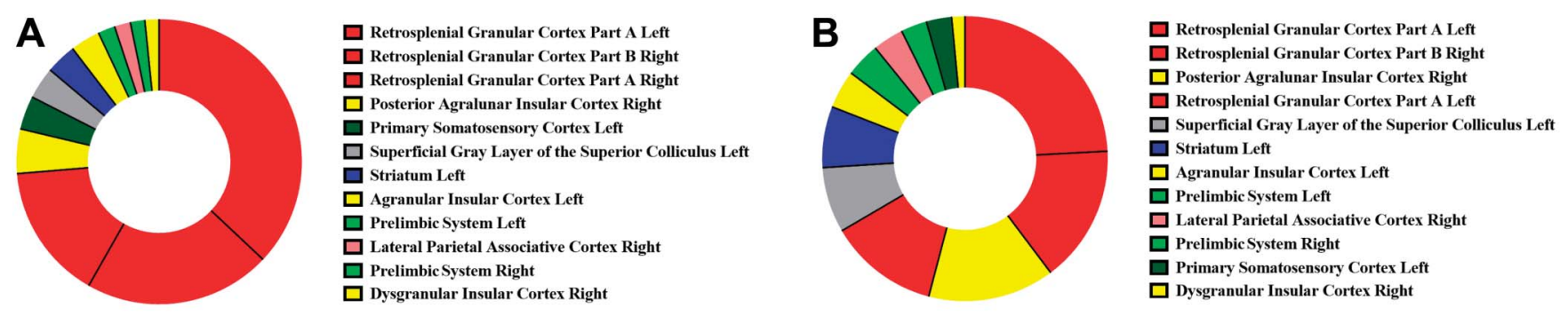
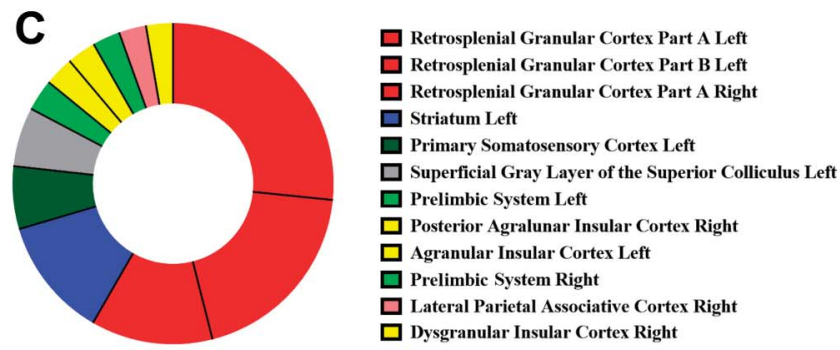

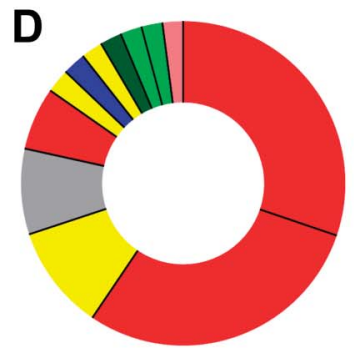

Figure 3. Centrality of common regions correlated with the pain scores. From the pain score correlation networks, we identified 12 systematic regions involved in neuropathic pain processes. Influence of each region in the $4 \mathrm{~g}$ von Frey (A), $8 \mathrm{~g}$ von Frey (B), $15 \mathrm{~g}$ von Frey (C), and acetone (D) networks was evaluated by calculating the degree of centrality of each network. Overall, for each network, the retrosplenial granular cortex displayed a strong centrality suggesting a crucial role of this region in central neuropathic pain processes. Centrality data were calculating using the Network-Based Statistic Tool Kit using 5000 permutations and an extended threshold set at $0.05(n=21)$.

showed that both measures allow to distinguish "neuropathic pain" to "no neuropathic pain" animals. Interestingly, even if the $\mathrm{RSgA}$-Prelimbic region measure had a better specificity (sensitivity $=0.455, \mathrm{Cl}=0.168-0.766$ and specificity $=0.9, \mathrm{Cl}$ $=0.555-0.998$, likelihood ratio = 6.364), the RSgA-Subiculum measure exhibited a better sensitivity to detect neuropathic pain (sensitivity $=0.546, \mathrm{Cl}=0.234-0.833$ and specificity $=0.889, \mathrm{Cl}$ $=0.518-0.997$, likelihood ratio $=4.909$ ).

\subsection{Morphometric modifications of some brain structures including the RSgA following neuropathic pain}

Using a VBM approach, we evaluated brain morphological modifications occurring in SNI animals compared with Sham. We observed a significant increase of GMC values in previously identified regions such as both left and right RSgA cortices, the dorsal PAG, the hippocampus, the ventro-postero-median nucleus of the thalamus, and the deep mesencephalic nucleus in SNI rats compared with Sham (Figs. 7A and C). Our analysis also revealed a significant decrease of GMC values within the primary somatosensory cortex Barrel field, the PtA, the dorsal part of the medial geniculate nucleus, and the dorsal and ventral part of the medial geniculate nucleus (Figs. 7A and C). To date, variation of GMC is generally interpreted as cell-size modification, neuronal, and/or glial cells genesis/apoptosis. ${ }^{4,29}$ Therefore, we investigated in the VBM animals, microglia and astrocytes activation, and the neuronal population in the RSgA, using immunohistochemistry. We observed a significant increase of Iba1 and GFAP positive cell density in SNI animals 2 months after surgery compared with Sham, suggesting microglia and astrocytes activation within the RSgA of neuropathic pain animals (Fig. 7B and representative photomicrographs). However, no significant modification of neuronal density in $\mathrm{SNI}$ animals has been observed (Fig. 7B). The RSgA GMC values were significantly increased in the 1- and 2-months SNI groups compared with their respective Sham groups (Fig. 7D). In this context, we tested the $\mathrm{ROC}$ procedure to estimate both sensitivity and specificity of RSgA's GMC values to predict neuropathic pain. The ROC analysis demonstrated that RSgA's GMC values allow to significantly distinguish "neuropathic pain" to "no neuropathic pain" animals with better sensitivity and specificity than those observed for the functional connectivity measures (sensitivity = $0.818, \mathrm{Cl}=0.482-0.977$, specificity $=0.9, \mathrm{Cl}=0.555-0.998$, likelihood ratio $=8,182$, Fig. 7E).

\section{Discussion}

\subsection{Brain connectome and morphology in neuropathic pain}

As reported in previous studies, the SNI model induces longlasting changes of both thermal and mechanical thresholds characterized by the development of allodynia and hyperalgesia in operated rats. ${ }^{3,24,37}$ We demonstrated that mechanical and thermal thresholds significantly correlated with the enhancement of a large number of functional connections through the entire brain. However, substantial differences between both mechanical and thermal allodynic networks have been found with some structures specific to the mechanical allodynic network. In addition, the mechanical hyperalgesic network (ie, $15 \mathrm{~g}$ Von Frey filament) involved a larger number of connections (92 functional connections) than the mechanical allodynic network (47 functional connections). Thus, like at the peripheral level where noxious stimulations are encoded by specific transduction mechanisms by primary afferent fibres, specific central networks exist within the brain to evaluate both modality and intensity of a noxious stimulation. Previous works in both healthy volunteers and neuropathic patients when exposed to allodynic or hyperalgesic brush, heat or cold stimulations, experienced pain in 

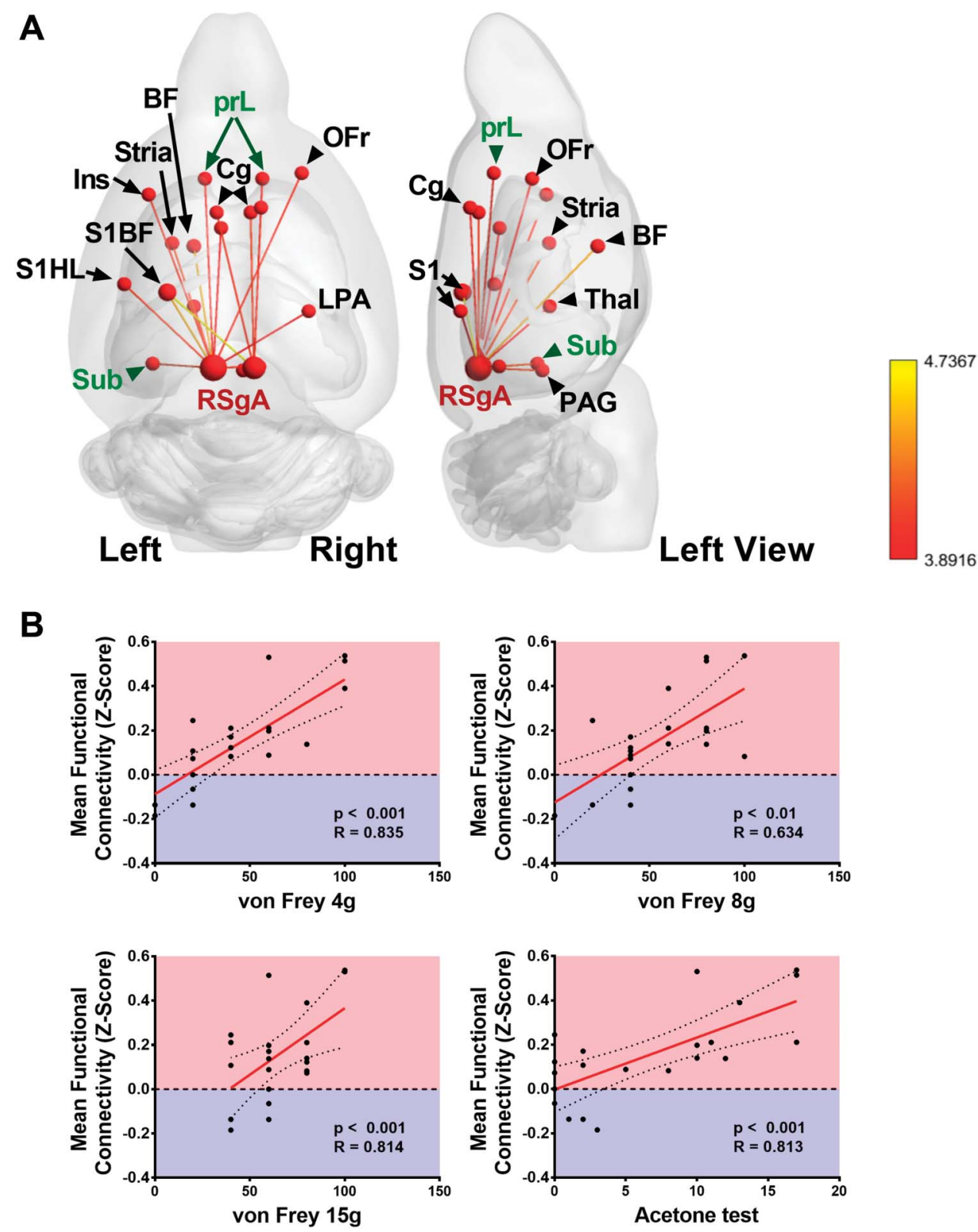

Figure 4. (A) Effect of spared nerve injury on RSgA functional connectivity. RSgA functional connectivity with the cingulate (Cg), insular (Ins), lateral parietal cortex (LPA), orbital frontal cortex (OFr), prelimbic system (prL), periaqueductal gray (PAG), somatosensory hind limb (S1HL) and barrel field (S1BF), striatum (Stria), and subiculum (Sub) were significantly correlated with $4 \mathrm{~g}$ von Frey, $8 \mathrm{~g}$ von Frey, $15 \mathrm{~g}$ von Frey, and acetone pain scores (B). Correlation was estimated using a two-tailed parametric Pearson test, and group comparison was estimated using a one-way ANOVA followed by Holm-Sidak post hoc test.

a similar manner but prompted distinct patterns of brain activation. ${ }^{64,65}$

A deeper investigation of the networks' topology showed that the global efficiency, transitivity, global clustering, and small worldness in SNI animals remained constant and similar to Sham in agreement with Baliki et al. $^{9}$ using the same model. These authors also observed functional connectivity modifications between Sham and SNI animals 28 days but not 5 days after $\mathrm{SNI}$ and demonstrated that neuropathic pain was associated with an alteration of the information transfer between the limbic and the nociceptive systems. Here, we found that the local metrics of several cortices such as the insula, the somatosensory, parietal, prelimbic, and retrosplenial regions as well as subcortical regions such as the gray layer of the superior colliculus and striatum displayed significant modifications of their centrality demonstrating that these regions promote important functional connections with the rest of the brain in chronic pain conditions. ${ }^{57}$ Surprisingly, all our functional analysis highlights the contralateral part $A$ of the retrosplenial granular cortex (RSgA).

Using a VBM approach, we observed that chronic pain significantly increased the mean GMC in several brain structures including the retrosplenial granular cortex part $A(R S g A)$. We also found a significant decrease of GMC within some structures such as the PtA and S1BF. Changes in the GMC of several brain regions in a chronic pain context has been already reported in numerous humans and some animal studies. ${ }^{11,66}$ To date, these mesoscopic GM modifications are still a matter of debate but are generally considered as cell size modification, neuronal or glial 


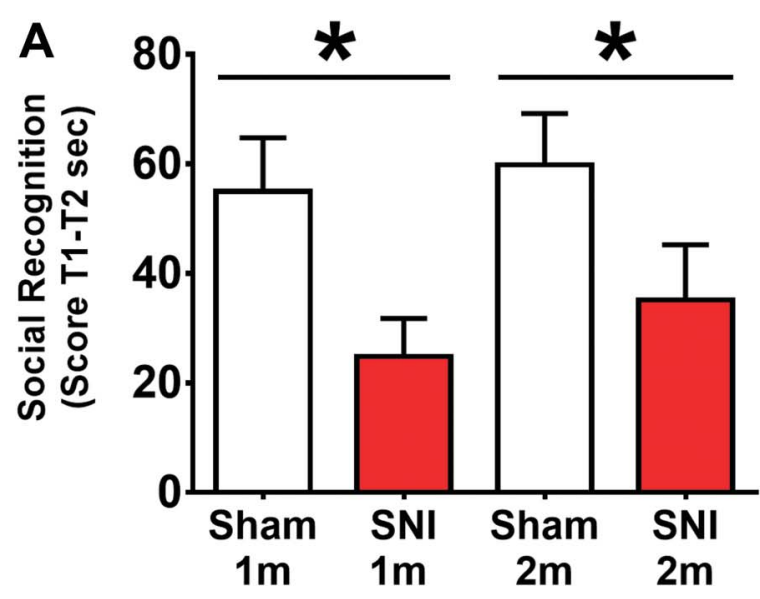

B

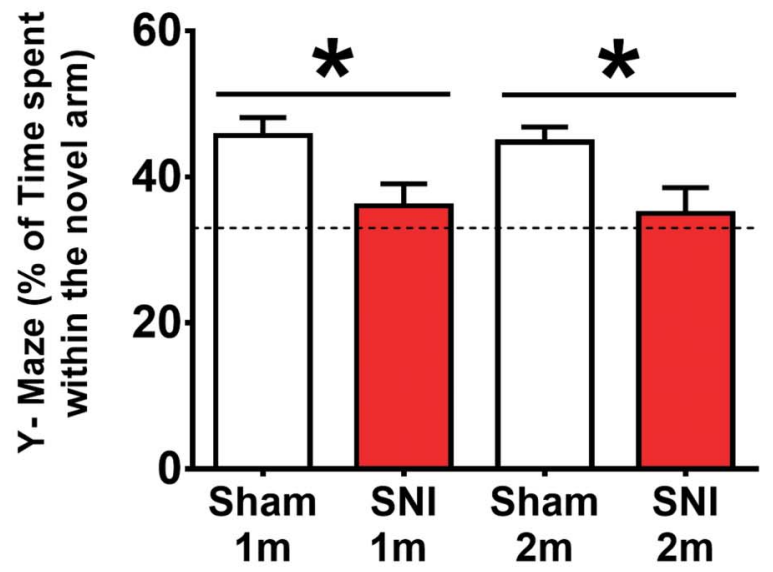

C

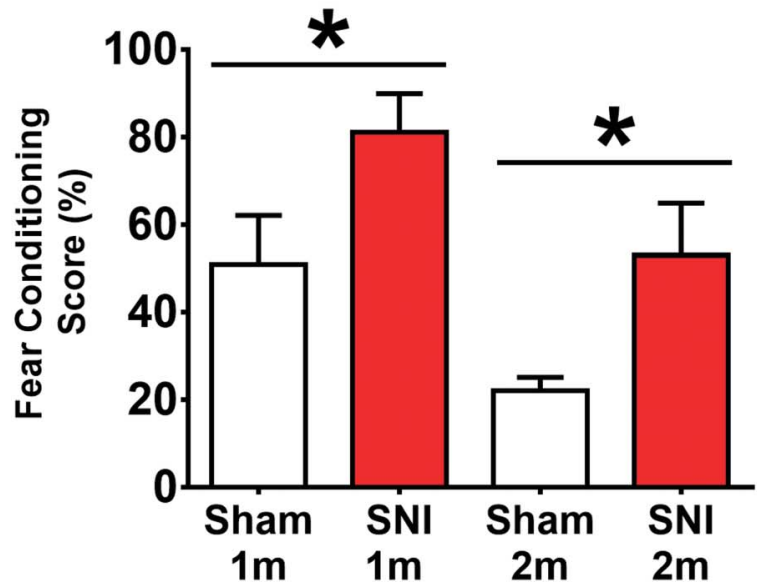

Figure 5. Influence of the Spared Nerve Injury on social/spatial memory and fear conditioning. We observed the development of a significant decrease of both social (A) and spatial (B) memory in SNI animals using the social recognition and the Y-Maze tests, respectively. (C) Moreover, we observed a significant increase of the response to fear in SNI animals using the fear conditioning test. Data were compared using on rank one-way ANOVA followed by Holm-Sidak post hoc test. Error bars represent the SEM. ${ }^{*} P<$ 0.05 compared with the Sham group ( $n=5-6$ per group and time point). ANOVA, analysis of variance; SNI, spared nerve injury. cells genesis/apoptosis, spine density, and/or blood flow modifications. ${ }^{4,29,74}$ Hence, structural modifications found with the VBM analysis suggest that cellular alterations sustain the functional alterations of SNI brain networks observed with the functional analysis notably in the retrosplenial cortices.

\subsection{The retrosplenial cortex as a pain integrator structure}

Our functional analysis of the contralateral RSgA connectivity revealed that it becomes an important integrative structure characterized by a significant increase of its functional connectivity with several structures. Thus, the contralateral RSgA in the SNI model seems an important hub, which integrates the nociceptive information and works directly with numerous painrelated structures in a segregated network. Other studies have demonstrated an increase of the cerebral blood flow, BOLD, and manganese-enhanced signals within the retrosplenial cortex in different pain models, ${ }^{10,26}$ suggesting that RSgA activation is conserved through different types of chronic pain. Moreover, aberrant DMN functioning in chronic pain patients has been reported with the functional connectivity between the PFC and the retrosplenial cortex (posterior cingulate) correlated with pain rumination, underlying their involvement in the emotional aspect of pain. ${ }^{8,32,40,45}$

Morphologically, our VBM study showed an increase of the RSgA GMC associated with a significant activation of astrocytes and microglial cells without any modification of neuronal density. Both brain astrocytes and microglial activation were already reported in a chronic pain context, but as far as our knowledge, never within the RSgA. ${ }^{27,59}$ Astrocytes activation has been found in the cingulate cortex after sciatic nerve ligation ${ }^{34,46}$ and in the nucleus of the tractus solitarii after colonic inflammation. ${ }^{67}$ The role of brain astrocytes and/or glial cells in chronic pain is still largely unknown, but evidences suggest that these cells act onto neuron metabolism and activation as regulators by tuning pronociceptive or antinociceptive mechanisms through neurotrophin and/or cytokine secretions, synapse maintenance, and neurotransmitters metabolisms. ${ }^{20}$ Interestingly, the deep morphological modifications within the contralateral RSgA are partially in agreement with Seminowicz et al., ${ }^{66}$ which demonstrated a significant decrease of the retrosplenial cortex volume 4 months after SNI using deformation-based morphometry approach. Hence, our VBM and histological results highlight subtle modifications of the GM within the retrosplenial cortex occurring early in the SNI model as well as modifications of functional connectivity.

Within the retrosplenial region, the RSgA is critically positioned between the hippocampal formation and the neocortex and has been involved in the integration of memory process, limbic information, and the integration of sensitive information conveyed by the thalamus and pain-related structures, ${ }^{21}$ supporting a central role of the RSgA in the integration of both sensory and emotional aspects of pain. Indeed, electrical stimulation of the $\mathrm{RSgA}$ produced antinociception in some pain tests and the incision pain model through the activation of specific descending inhibitory pathways. ${ }^{54,56}$ Interestingly, the functional connectivity of the RSgA with the prelimbic system and the subiculum have been previously reported to be associated with fear and mnesic impairments in rodents, respectively. $35,51,52,68$ Therefore, we investigated the development of cognitive comorbidities in Sham and SNI animals. ${ }^{49}$ Using appropriate tests, we observed both social and spatial memory impairments associated with an 
A

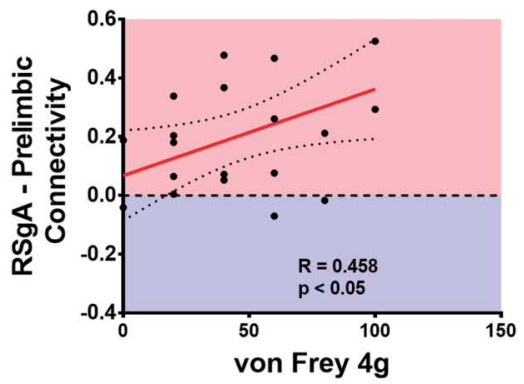

B

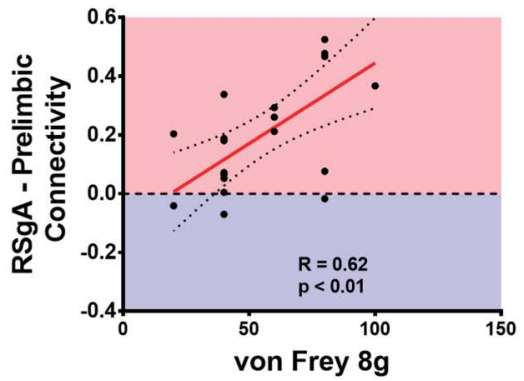

C

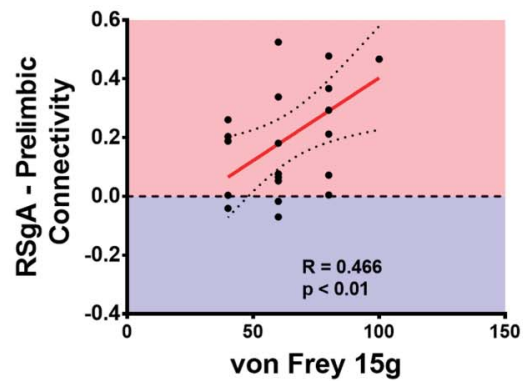

D

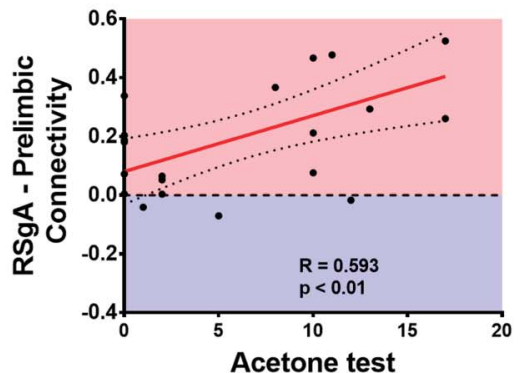

$\mathbf{E}$

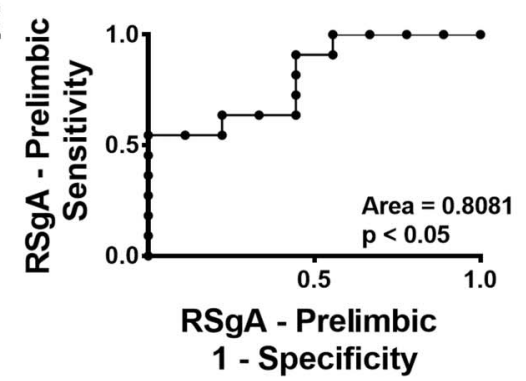

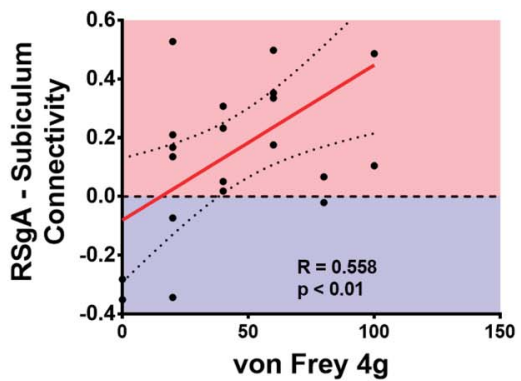
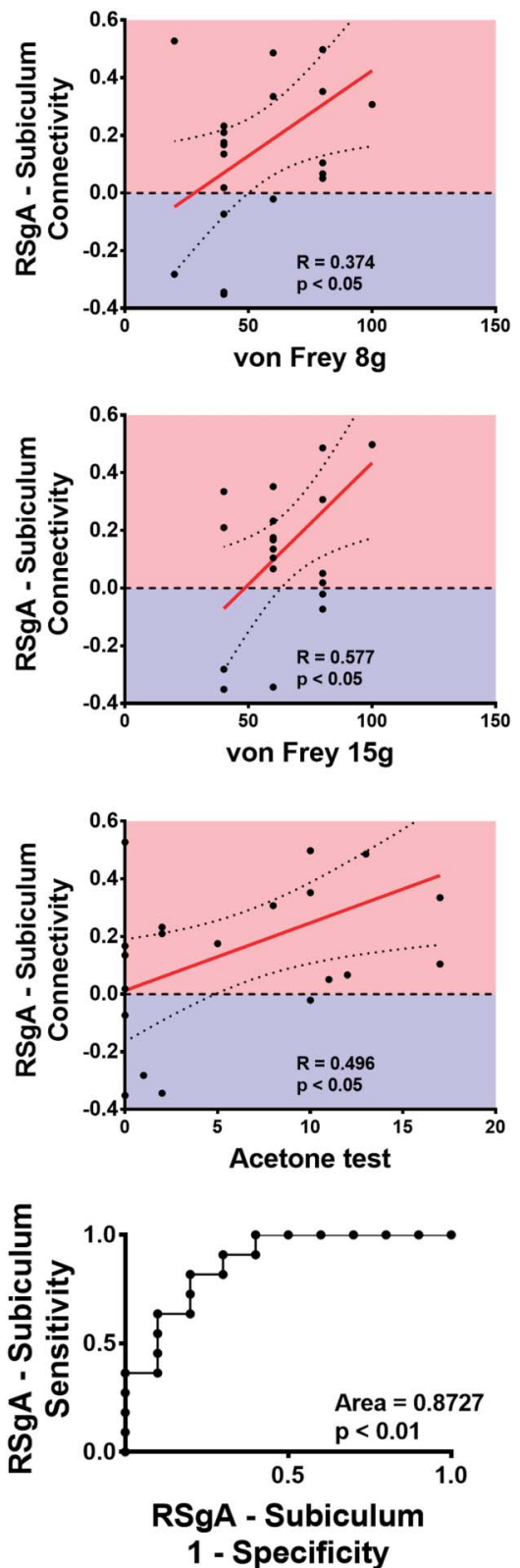

Figure 6. RSgA, prelimbic, and RSgA, subiculum functional connectivity, as a pain imaging biomarker. RSgA, prelimbic (left panel), and RSgA, subiculum (right panel) functional connectivity, were significantly correlated with $4 \mathrm{~g}$ von Frey (A), $8 \mathrm{~g}$ von Frey (B), $15 \mathrm{~g}$ von Frey (C), and acetone (D) pain scores. ROC curves analysis of Pearson coefficient of RSgA, prelimbic (left panel), and subiculum (right panel) connections, between Sham and SNI animals showed that both measures could be good predictors of neuropathic pain (E). Correlation were estimated using a two-tailed parametric Pearson test $(n=21)$. SNI, spared nerve injury.

increase response to fear, suggesting cognitive and emotional impairments in SNI animals. Changes of RSgA connectivity with the prelimbic system and the subiculum could be related to the development of pain comorbidities such as cognitive impairments and response to fear. In addition, these changes are significantly correlated with peripheral pain scores providing indirect evidence of RSgA involvement in the cognitive-emotional aspect of pain. Interestingly, both RSgA, prelimbic, and RSgA, 


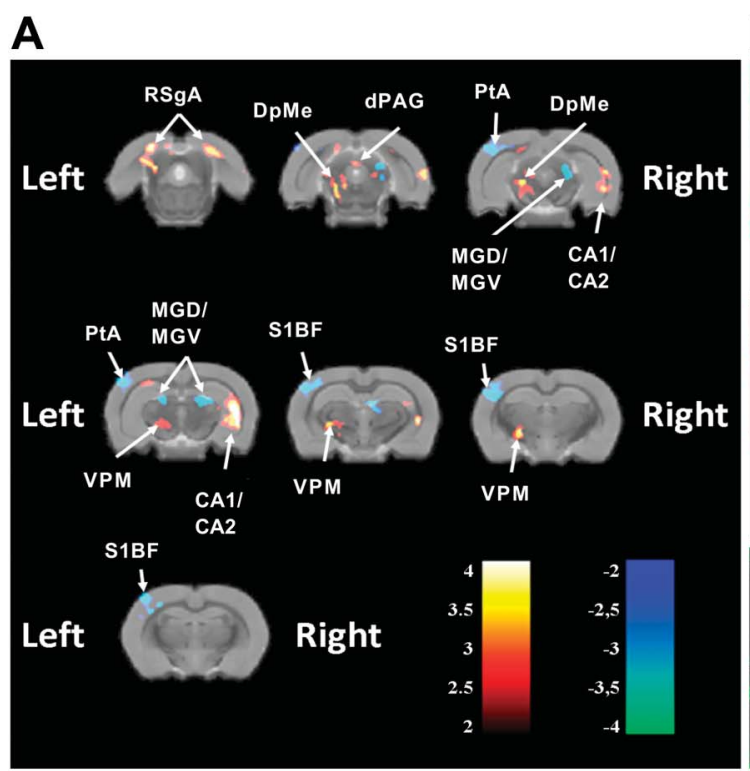

Regions Of Interest $T$ value $Z$ value pvalue (Uncorrected)

\begin{tabular}{lccc}
\hline RSgA Right & 4,3 & 3,52 & $>0,001$ \\
RSgA Left & 4,48 & 3,62 & $>0,001$ \\
DpME Left & 2,53 & 2,31 & 0,01 \\
dPAG & 3,97 & 3,32 & $>0,001$ \\
VPM Left & 2,69 & 2,44 & 0,007 \\
CA1/CA2 & 9,76 & 5,69 & $>0,001$ \\
PtA Left & $-4,2$ & $-3,46$ & $>0,001$ \\
MGD/MGV & $-7,54$ & -5 & $>0,001$ \\
S1BF Left & $-3,82$ & $-3,22$ & 0,001 \\
\hline
\end{tabular}
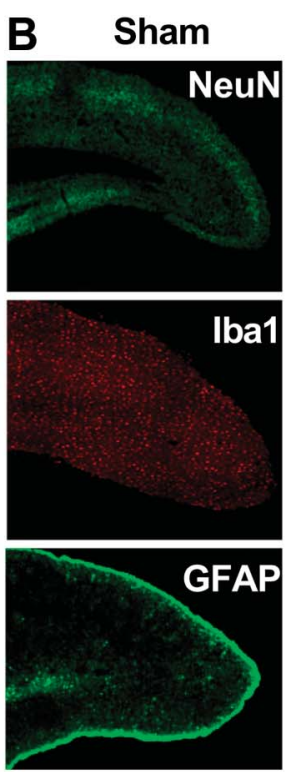

D

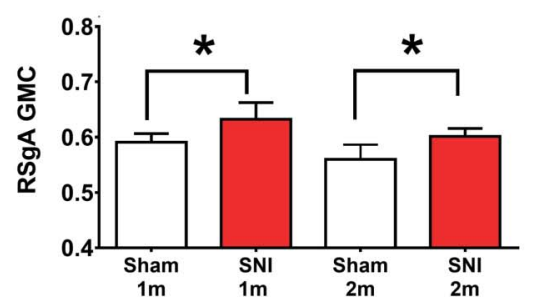

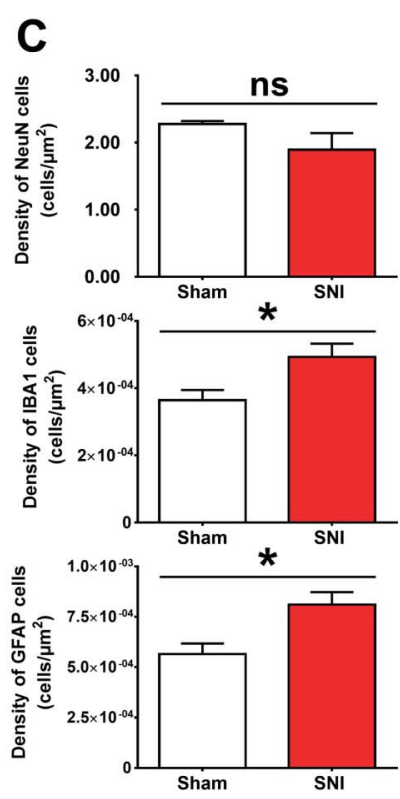

E

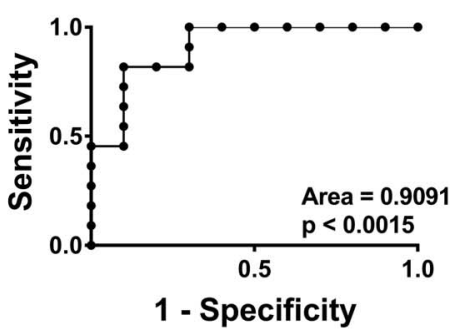

Figure 7. Effect of spared nerve injury on brain morphometry and local RSgA glial activation and neuronal density. (A) Voxel-based morphology (VBM) analysis demonstrated a significant increase of gray matter concentration (GMC, red scale, and table below) within the retrosplenial granular cortex part A (RSgA), deep mesencephalic nucleus (DpME), dorsal periaqueductal gray substance (dPAG), region 1 and 2 of the cornus ammonis (CA1/CA2), and in the ventro-posteromedian nucleus of the thalamus (VPM). We also observed a significant decrease of GMC (blue scale) within the parietal associative cortex (PtA), dorsal part of the medial geniculate nucleus (MGD), dorsal and ventral part of the medial geniculate nucleus (MGD/MGV), and in the somatosensory cortex, barrel field part (S1BF). (B) Representative photomicrographs of NeuN-, Iba1-, and GFAP-positive cells within the retrosplenial cortex in both Sham and SNI animals. (C) Quantification of Iba1- and GFAP-positive cells within the retrosplenial cortex showed a significant increase of both microglia and astrocytic cells in SNI animals compared with Sham while neuronal density was unaffected (histogram). (D) Gray matter concentration values within the RSgA 1 and 2 months after SNI group showing a significant increase of GMC values in the SNI group compared with Sham. (E) ROC curve demonstrated that RSgA GMC could be a good predictor of neuropathic pain. In (A), data are represented as a t-score map for $t_{(19)}=2.213$, (FDR-corrected, $P<0.02,200$ voxels, $n=21$, peak values). In (C), group comparison was estimated using a two-tailed Mann-Whitney U test. ${ }^{*} P<0.05$, compared with the Sham group ( $\left.n=21\right)$. In (D), data were compared using ANOVA followed by Holm-Sidak post hoc test. Error bars represent the SEM. ${ }^{*} P<0.05$, compared with the Sham group $(n=21)$. ANOVA, analysis of variance; SNI, spared nerve injury.

subiculum functional connectivity scores, allowed to predict neuropathic pain. Using an ROC procedure to estimate the sensitivity (ability to detect "neuropathic pain") and the specificity (ability to detect "no neuropathic pain") of each functional connection, we found a significant predictive score for both measures. Moreover, the RSgA GMC values have also been found to be a significant predictive score of painful animals. Taken together, our study strongly supports a critical role of the RSgA in both sensory and cognitive-emotional aspects of neuropathic pain.

There are some limitations in our study. First, we used a crosssectional design comparing 2 different groups of rats after surgery, which may reduce the statistical effect size compared with a longitudinal design. Anesthesia has also been reported to alter functional connectivity between brain regions. ${ }^{47}$ Nonetheless, some reports indicated that the rs-fMRI signal in isoflurane anesthetized rats is mostly of neural origin, ${ }^{39}$ and that functional connectivity of brain networks preserves topological properties. ${ }^{38}$ Performing imaging studies in awake animals avoids possible anesthetic confounds but raises new concerns, particularly those related to stress and motions. Our study also suggests the RsgA as a potential biomarker of neuropathic pain. We think that it should be rather considered as a part of a neurological pain signature sensitive and probably specific to chronic pain, involving other brain regions (ie, somatosensory, cingulate and insular cortices, as well as thalamus and PAG). ${ }^{69,70}$ However, these "pain signature" models must face further test validity across the different phases in pain development and across different pain etiologies, and challenged by pharmacological studies.

\section{Conclusion}

Here, we brought evidences that the RSgA is a critical hub, which could integrate both sensory and cognitive-emotional aspects of neuropathic pain in a segregated network centred on this structure. This cortex was also impacted morphologically and exhibited glial activation following neuropathic pain. Importantly, 
we demonstrated that both brain functional and morphological $\mathrm{MRI}$ metrics of the RSgA could be used as a possible objective imaging biomarker to discriminate painful to nonpainful animals. Further studies deciphering pathophysiological mechanisms occurring in the RSgA and its involvement during chronic pain could help in its understanding and management.

\section{Conflict of interest statement}

The authors have no conflict of interest to declare.

\section{Acknowledgments}

This work was supported by Inserm, Clermont Auvergne University, grants from Agence Nationale de la Recherche (ANR13-BSV1-0006-03), the Société Française d'Étude et de Traitement de la Douleur.

\section{Appendix A. Supplemental digital content}

Supplemental digital content associated with this article can be found online at http://links.lww.com/PAIN/A805, http://links.Iww. com/PAIN/A806, http://links.Iww.com/PAIN/A807, http://links. Iww.com/PAIN/A808, http://links.Iww.com/PAIN/A809, and http://links.Iww.com/PAIN/A811.

\section{Article history:}

Received 13 February 2019

Received in revised form 8 April 2019

Accepted 2 May 2019

Available online 22 May 2019

\section{References}

[1] Apkarian AV, Bushnell MC, Treede RD, Zubieta JK. Human brain mechanisms of pain perception and regulation in health and disease. Eur J Pain 2005;9:463-84.

[2] Apkarian AV, Hashmi JA, Baliki MN. Pain and the brain: specificity and plasticity of the brain in clinical chronic pain. PAIN 2011;152:S49-64.

[3] Apkarian AV, Mutso AA, Centeno MV, Kan L, Wu M, Levinstein M, Banisadr G, Gobeske KT, Miller RJ, Radulovic J, Hen R, Kessler JA. Role of adult hippocampal neurogenesis in persistent pain. PAIN 2016;157: 418-28.

[4] Arentsen T, Qian Y, Gkotzis S, Femenia T, Wang T, Udekwu K, Forssberg H, Diaz Heijtz R. The bacterial peptidoglycan-sensing molecule Pglyrp2 modulates brain development and behavior. Mol Psychiatry 2017;22: 257-66.

[5] Ashburner J. A fast diffeomorphic image registration algorithm. Neuroimage 2007;38:95-113.

[6] Ashburner J, Friston KJ. Unified segmentation. Neuroimage 2005;26: 839-51.

[7] Ashburner J, Friston KJ. Voxel-based morphometry-the methods. Neuroimage 2000;11:805-21.

[8] Baliki MN, Baria AT, Apkarian AV. The cortical rhythms of chronic back pain. J Neurosci 2011;31:13981-90.

[9] Baliki MN, Chang PC, Baria AT, Centeno MV, Apkarian AV. Resting-sate functional reorganization of the rat limbic system following neuropathic injury. Sci Rep 2014;4:6186.

[10] Becerra L, Bishop J, Barmettler G, Kainz V, Burstein R, Borsook D. Brain network alterations in the inflammatory soup animal model of migraine. Brain Res 2017;1660:36-46.

[11] Bilbao A, Falfán-Melgoza C, Leixner S, Becker R, Singaravelu SK, Sack M, Sartorius A, Spanagel R, Weber-Fahr W. Longitudinal structural and functional brain network alterations in a mouse model of neuropathic pain. Neuroscience 2018;387:104-15.

[12] Boly M, Faymonville ME, Schnakers C, Peigneux P, Lambermont B, Phillips C, Lancellotti P, Luxen A, Lamy M, Moonen G, Maquet P, Laureys S. Perception of pain in the minimally conscious state with PET activation: an observational study. Lancet Neurol 2008;7:1013-20.
[13] Borsook D, Becerra L. CNS animal fMRI in pain and analgesia. Neurosci Biobehav Rev 2011;35:1125-43.

[14] Borsook D, Becerra L, Hargreaves R. A role for fMRI in optimizing CNS drug development. Nat Rev Drug Discov 2006;5:411-24.

[15] Choi Y, Yoon YW, Na HS, Kim SH, Chung JM. Behavioral signs of ongoing pain and cold allodynia in a rat model of neuropathic pain. PAIN 1994;59:369-76.

[16] Colloca L, Ludman T, Bouhassira D, Baron R, Dickenson AH, Yarnitsky D, Freeman R, Truini A, Attal N, Finnerup NB, Eccleston C, Kalso E, Bennett DL, Dworkin RH, Raja SN. Neuropathic pain. Nat Rev Dis Primers 2017;3: 17002.

[17] Decosterd I, Woolf CJ. Spared nerve injury: an animal model of persistent peripheral neuropathic pain. PAIN 2000;87:149-58.

[18] Dellu F, Mayo W, Cherkaoui J, Le Moal M, Simon H. A two-trial memory task with automated recording: study in young and aged rats. Brain Res 1992;588:132-9.

[19] Flatters SJ, Bennett GJ. Studies of peripheral sensory nerves in paclitaxel-induced painful peripheral neuropathy: evidence for mitochondrial dysfunction. PAIN 2006;122:245-57.

[20] Gosselin RD, Suter MR, Ji RR, Decosterd I. Glial cells and chronic pain. Neuroscientist 2010;16:519-31.

[21] van Groen T, Wyss JM. Connections of the retrosplenial granular a cortex in the rat. J Comp Neurol 1990;300:593-606.

[22] Hashmi JA, Kong J, Spaeth R, Khan S, Kaptchuk TJ, Gollub RL. Functional network architecture predicts psychologically mediated analgesia related to treatment in chronic knee pain patients. J Neurosci 2014;34:3924-36.

[23] Henry DE, Chiodo AE, Yang W. Central nervous system reorganization in a variety of chronic pain states: a review. PM R 2011;3:1116-25.

[24] Hubbard CS, Khan SA, Xu S, Cha M, Masri R, Seminowicz DA. Behavioral, metabolic and functional brain changes in a rat model of chronic neuropathic pain: a longitudinal MRI study. Neuroimage 2015; 107:333-44.

[25] lannetti GD, Mouraux A. From the neuromatrix to the pain matrix (and back). Exp Brain Res 2010;205:1-12.

[26] Jeong $\mathrm{KY}$, Kang JH. Investigation of spinal nerve ligation-mediated functional activation of the rat brain using manganese-enhanced MRI. Exp Anim 2018;67:23-9.

[27] Ji RR, Chamessian A, Zhang YQ. Pain regulation by non-neuronal cells and inflammation. Science 2016;354:572-7.

[28] Keifer OP Jr, Hurt RC, Gutman DA, Keilholz SD, Gourley SL, Ressler KJ. Voxel-based morphometry predicts shifts in dendritic spine density and morphology with auditory fear conditioning. Nat Commun 2015;6: 7582.

[29] Kelly JR, Kennedy PJ, Cryan JF, Dinan TG, Clarke G, Hyland NP. Breaking down the barriers: the gut microbiome, intestinal permeability and stress-related psychiatric disorders. Front Cell Neurosci 2015;9: 392.

[30] Kelly RE, Alexopoulos GS, Wang Z, Gunning FM, Murphy CF, Morimoto SS, Kanellopoulos D, Jia Z, Lim KO, Hoptman MJ. Visual inspection of independent components: defining a procedure for artifact removal from fMRI data. J Neurosci Methods 2010;189:233-45.

[31] Kucyi A, Davis KD. The dynamic pain connectome. Trends Neurosci 2015;38:86-95.

[32] Kucyi A, Moayedi M, Weissman-Fogel I, Goldberg MB, Freeman BV, Tenenbaum HC, Davis KD. Enhanced medial prefrontal-default mode network functional connectivity in chronic pain and its association with pain rumination. J Neurosci 2014;34:3969-75.

[33] Kunde V, Treede RD. Topography of middle-latency somatosensory evoked potentials following painful laser stimuli and non-painful electrical stimuli. Electroencephalogr Clin Neurophysiol 1993;88:280-9.

[34] Kuzumaki N, Narita M, Narita M, Hareyama N, Niikura K, Nagumo Y, Nozaki H, Amano T, Suzuki T. Chronic pain-induced astrocyte activation in the cingulate cortex with no change in neural or glial differentiation from neural stem cells in mice. Neurosci Lett 2007;415:22-7.

[35] Kwapis JL, Jarome TJ, Lee JL, Helmstetter FJ. The retrosplenial cortex is involved in the formation of memory for context and trace fear conditioning. Neurobiol Learn Mem 2015;123:110-16.

[36] Leite-Almeida H, Almeida-Torres L, Mesquita AR, Pertovaara A, Sousa N, Cerqueira JJ, Almeida A. The impact of age on emotional and cognitive behaviours triggered by experimental neuropathy in rats. PAIN 2009;144: 57-65.

[37] Leite-Almeida H, Cerqueira JJ, Wei H, Ribeiro-Costa N, Anjos-Martins H, Sousa N, Pertovaara A, Almeida A. Differential effects of left/right neuropathy on rats' anxiety and cognitive behavior. PAIN 2012;153: 2218-25.

[38] Liang Z, King J, Zhang N. Intrinsic organization of the anesthetized brain. J Neurosci 2012;32:10183-91. 
[39] Liu X, Zhu XH, Zhang Y, Chen W. Neural origin of spontaneous hemodynamic fluctuations in rats under burst-suppression anesthesia condition. Cereb Cortex 2011;21:374-84.

[40] Loggia ML, Kim J, Gollub RL, Vangel MG, Kirsch I, Kong J, Wasan AD, Napadow V. Default mode network connectivity encodes clinical pain: an arterial spin labeling study. PAIN 2013;154:24-33.

[41] Lui F, Duzzi D, Corradini M, Serafini M, Baraldi P, Porro CA. Touch or pain? Spatio-temporal patterns of cortical fMRI activity following brief mechanical stimuli. PAIN 2008;138:362-74.

[42] Magalhães R, Barrière DA, Novais A, Marques F, Marques P, Cerqueira J, Sousa JC, Cachia A, Boumezbeur F, Bottlaender M, Jay TM, Mériaux S, Sousa N. The dynamics of stress: a longitudinal MRI study of rat brain structure and connectome. Mol Psychiatry 2017;23:1998-2006.

[43] Miller G. Neuroscience. Brain scans of pain raise questions for the law. Science 2009;323:195.

[44] Mutso AA, Radzicki D, Baliki MN, Huang L, Banisadr G, Centeno MV, Radulovic J, Martina M, Miller RJ, Apkarian AV. Abnormalities in hippocampal functioning with persistent pain. J Neurosci 2012;32: 5747-56.

[45] Napadow V, Kim J, Clauw DJ, Harris RE. Decreased intrinsic brain connectivity is associated with reduced clinical pain in fibromyalgia. Arthritis Rheum 2012;64:2398-403.

[46] Narita M, Kuzumaki N, Narita M, Kaneko C, Hareyama N, Miyatake M, Shindo K, Miyoshi K, Nakajima M, Nagumo Y, Sato F, Wachi H, Seyama $Y$, Suzuki T. Chronic pain-induced emotional dysfunction is associated with astrogliosis due to cortical delta-opioid receptor dysfunction. J Neurochem 2006;97:1369-78.

[47] Paasonen J, Stenroos P, Salo RA, Kiviniemi V, Gröhn O. Functional connectivity under six anesthesia protocols and the awake condition in rat brain. Neuroimage 2018;172:9-20.

[48] Papp EA, Leergaard TB, Calabrese E, Johnson GA, Bjaalie JG. Waxholm space atlas of the Sprague dawley rat brain. Neuroimage 2014;97: 374-86.

[49] Percie du Sert N, Rice ASC. Improving the translation of analgesic drugs to the clinic: animal models of neuropathic pain. Br J Pharmacol 2014; 171:2951-63.

[50] Perio A, Terranova JP, Worms P, Bluthe RM, Dantzer R, Biziere K. Specific modulation of social memory in rats by cholinomimetic and nootropic drugs, by benzodiazepine inverse agonists, but not by psychostimulants. Psychopharmacology (Berl) 1989;97:262-8.

[51] Powell AL, Nelson AJD, Hindley E, Davies M, Aggleton JP, Vann SD. The rat retrosplenial cortex as a link for frontal functions: a lesion analysis. Behav Brain Res 2017;335:88-102.

[52] Powell AL, Vann SD, Olarte-Sánchez CM, Kinnavane L, Davies M, Amin E, Aggleton JP, Nelson AJD. The retrosplenial cortex and object recency memory in the rat. Eur J Neurosci 2017;45:1451-64.

[53] Reddan MC, Wager TD. Modeling pain using fMRI: from regions to biomarkers. Neurosci Bull 2018;34:208-15.

[54] Reis GM, Dias QM, Silveira JWS, Del Vecchio F, Garcia-Cairasco N, Prado WA. Antinociceptive effect of stimulating the occipital or retrosplenial cortex in rats. J Pain 2010;11:1015-26.

[55] Ren WJ, Liu Y, Zhou LJ, Li W, Zhong Y, Pang RP, Xin WJ, Wei XH, Wang J, Zhu HQ, Wu CY, Qin ZH, Liu G, Liu XG. Peripheral nerve injury leads to working memory deficits and dysfunction of the hippocampus by upregulation of TNF- $\alpha$ in rodents. Neuropsychopharmacology 2011;36: 979-92.

[56] Rossaneis AC, Reis GM, Prado WA. Stimulation of the occipital or retrosplenial cortex reduces incision pain in rats. Pharmacol Biochem Behav 2011;100:220-7

[57] Rubinov M, Sporns O. Complex network measures of brain connectivity: uses and interpretations. Neuroimage 2010;52:1059-69.

[58] Salomons TV, lannetti GD, Liang M, Wood JN. The "pain matrix" in painfree individuals. JAMA Neurol 2016;73:755-6.

[59] Salter MW, Stevens B. Microglia emerge as central players in brain disease. Nat Med 2017;23:1018-27.

[60] Sawiak SJ, Picq JL, Dhenain M. Voxel-based morphometry analyses of in vivo MRI in the aging mouse lemur primate. Front Aging Neurosci 2014;6:82.

[61] Sawiak SJ, Wood NI, Williams GB, Morton AJ, Carpenter TA. SPMMouse: a new toolbox for SPM in the animal brain. 17th Meeting of the Int'I Soc Mag Res in Med. Hawaii, USA 2009. p 1086.

[62] Sawiak SJ, Wood NI, Williams GB, Morton AJ, Carpenter TA. Voxelbased morphometry with templates and validation in a mouse model of Huntington's disease. Magn Reson Imaging 2013;31:1522-31.

[63] Schweinhardt P, Bountra C, Tracey I. Pharmacological FMRI in the development of new analgesic compounds. NMR Biomed 2006;19:702-11.

[64] Seifert F, Jungfer I, Schmelz M, Maihöfner C. Representation of UV-Binduced thermal and mechanical hyperalgesia in the human brain: a functional MRI study. Hum Brain Mapp 2008;29:1327-42.

[65] Seifert F, Maihöfner C. Representation of cold allodynia in the human brain-a functional MRI study. Neuroimage 2007;35:1168-80.

[66] Seminowicz DA, Laferriere AL, Millecamps M, Yu JSC, Coderre TJ, Bushnell MC. MRI structural brain changes associated with sensory and emotional function in a rat model of long-term neuropathic pain. Neuroimage 2009;47:1007-14.

[67] Sun YN, Luo JY, Rao ZR, Lan L, Duan L. GFAP and Fos immunoreactivity in lumbo-sacral spinal cord and medulla oblongata after chronic colonic inflammation in rats. World J Gastroenterol 2005;11:4827-32.

[68] Todd TP, Bucci DJ. Retrosplenial cortex and long-term memory: molecules to behavior. Neural Plast 2015;2015:414173.

[69] Valdes-Hernandez PA, Sumiyoshi A, Nonaka H, Haga R, Aubert-Vasquez E, Ogawa T, Iturria-Medina Y, Riera JJ, Kawashima R. An in vivo MRI template set for morphometry, tissue segmentation, and fMRI localization in rats. Front Neuroinform 2011;5:26.

[70] Wager TD, Atlas LY, Lindquist MA, Roy M, Woo CW, Kross E. An fMRIbased neurologic signature of physical pain. N Engl J Med 2013;368: 1388-97.

[71] Whitfield-Gabrieli S, Nieto-Castanon A. Conn: a functional connectivity toolbox for correlated and anticorrelated brain networks. Brain Connect 2012;2:125-41.

[72] Xia M, Wang J, He Y. BrainNet viewer: a network visualization tool for human brain connectomics. PLoS One 2013;8:e68910.

[73] Zalesky A, Fornito A, Bullmore ET. Network-based statistic: identifying differences in brain networks. Neuroimage 2010;53:1197-207.

[74] Zheng L, Cleppien D, Gass N, Falfan-Melgoza C, Vollmayr B, Hesser J, Weber-Fahr W, Sartorius A. Influence of regional cerebral blood volume on voxel-based morphometry. NMR Biomed 2016;29:787-95.

[75] Zimmermann M. Ethical guidelines for investigations of experimental pain in conscious animals. PAIN 1983;16:109-10. 